\author{
Nermin Vučelj \\ Université de Niš, Faculté de philosophie \\ e-mail :nermin.vucelj@filfak.ni.ac.rs
}

\title{
LE PHILOSOPHE DIDEROT CONTRE L’ABBÉ DIDEROT ${ }^{1}$
}

Résumé : La présente recherche a pour dessein d'offrir une analyse de la polémique critique au sujet du religieux et de la tolérance dont les acteurs sont les frères Diderot, le philosophe Denis et l'abbé Didier-Pierre. Si nous partons de l'hypothèse que l'abbé Diderot était son lecteur désiré, voire un récipient effectif, le philosophe Diderot écrivait alors ses ouvrages visant son frère, ennemi de l'Encyclopédie et de la philosophie. Dans l'esprit de son encyclopédisme, l'aîné a vu son cadet comme le prototype du dévot intolérant qu'il devait éclairer. En revanche, le cadet a critiqué son aîné comme un irréligieux qui séparait, sans aucun fondement, la morale du christianisme. Le présent article tente de reconsidérer les positions de deux débatteurs à la lumière des arguments échangés.

Mots-clés : philosophe, abbé, religion, dévot, raison, Lumières, tolérance.

\section{Lumières de la raison contre Lumière du Ciel}

Dans son étude La philosophie des Lumières (Die Philosophie der Aufklärung, 1932), Ernest Cassirer a conclu que l'essence même de la philosophie française des Lumières était «l'attitude critique et sceptique à l'égard de la religion », en soulignant que ce n'était point le trait caractéristique des Lumières allemandes et anglaises. ${ }^{2}$ La conclusion du philosophe allemand est bien justifiée si nous nous référons aux Lettres philosophiques de Voltaire, écrites pendant son séjour de trois ans (1726-1729) en Angleterre, car le Patriarche des philosophes a vu le royaume d'outre-Manche comme le pays de la liberté où le Parlement réglait le pouvoir du monarque, la philosophie étudiait les faits à la lumières de la raison et les religions coexistaient en paix.

Dans son article sur les Lumières françaises, Monique Cottret nous rappelle que la monarchie française a embastillé Voltaire, a emprisonné Diderot à Vincennes, a contraint Rousseau à l'exil, et conclut que les philosophes français n'ont jamais accédé à une telle reconnaissance en France comme les penseurs anglais dans leur patrie, par exemple John Locke étant considéré comme l'inspirateur du modèle politique de son pays. ${ }^{3}$ L'Angleterre avait donc une grande supériorité sur la France de la Révocation de l'Édit de Nantes et des dragonnades empruntons ici les mots à Laurent Versini, le dix-huitiémiste qui constate qu'après le séjour du philosophe français en Angleterre «le programme de Voltaire et celui des Lumières » sont désormais définis : lutte contre toutes les tyrannies du système, contre l'intolérance sociale et le

1 Cet article est rédigé dans le cadre du projet scientifique international Les langues, les littératures et les cultures romanes et slaves en contact et en divergence, $\mathrm{N}^{\mathrm{o}} 1001-13-01$, approuvé le $1^{\mathrm{er}}$ mars 2021 par la Faculté de Philosophie de l'Université de Niš.

2 Cassirer 1983, 153.

3 Cottret 1991, 437. 
fanatisme religieux. ${ }^{4}$ Ainsi, à la différence des Lumières à l'étranger, les Lumières en France se distinguent-elles par leur aspect frondeur et par l'antichristianisme de certains des philosophes.

L'encyclopédisme français, ce qui est l'appellation que Cassirer utilise pour les Lumières françaises, part ainsi « en guerre ouverte contre la religion, contre sa validité, contre sa prétendue vérité ». ${ }^{5}$ Le rôle de l'Encyclopédie y est incontournable, et son directeur Denis Diderot, selon Jean Ehrard, représente « la plus complète incarnation de l'esprit militant des Lumières, le Philosophe par excellence $»{ }^{6}$ Comme le rappelle Alain Viala, l'Encyclopédie avait une triple fonction : elle jouait le rôle de vulgarisation des connaissances, en s'adressant aux couches moyennes de la société ; elle affichait une fonction d'utilité pratique, en s'annonçant en sous-titre comme un Dictionnaire raisonné des sciences, des arts et des métiers; elle avait une tonalité militante contre les préjugés et les dogmes religieux en général. ${ }^{7}$

L'opposition entre la Raison et la Foi, à savoir entre le philosophe et le dévot, s'inscrit dans la notion même de Lumières. Comme l'a noté Monique Cottret, le XVIII ${ }^{\mathrm{e}}$ siècle a renversé la perspective du terme lumière, d'abord attribut de la toute-puissance divine : passée au pluriel et détachée de la métaphysique, ce substantif désigne désormais ce qui est propre à l'homme qui analyse et raisonne. ${ }^{8}$ Dans l'article encyclopédique "Philosophe », habituellement attribué à Dumarsais, il est écrit que la raison est si essentielle au philosophe comme la grâce divine est essentielle au dévot. ${ }^{9}$ Diderot reprend cette idée dans son ouvrage écrit pour l'impératrice russe Catherine II, Observation sur le Nakaz : les philosophes parlent au nom de la Raison, alors que les prêtres parlent au nom de Dieu. La religion ne sert que de prétexte au clergé pour masquer ses desseins. Ainsi, les Lumières de la raison s'opposent-elles à la Lumière du Ciel ; or, «la raison est l'ennemie de la foi $\gg .{ }^{10}$

Denis Diderot tente de persuader l'impératrice russe que la religion en tant qu'appui finit toujours par renverser la maison et que la distance entre l'autel et le trône ne peut jamais être trop grande. La maison menacée de fanatisme religieux dont parle Diderot est la société, mais, dans le cas de notre philosophe, c'est aussi la maison au sens propre, celui de la famille, en l'occurrence les Diderot, car les deux frères se sont opposés en ennemi : l'aîné, Denis, en tant que philosophe, et le cadet, Didier-Pierre, en tant qu'abbé.

\title{
Les frères ennemis
}

Comme l'a noté Arthur Wilson, Denis Diderot sortait d'un milieu intimement proche de la tradition de l'Église : ces deux oncles maternels étaient prêtres (l'un chanoine de la cathédrale, l'autre curé à Chassigny), tout comme les deux oncles de sa mère, qui étaient curés de campagne ; son oncle paternel était moine dominicain. ${ }^{11}$ De quatre enfants de Didier Diderot (couturier de Langres) et d'Angélique Vigneron (issue d'une famille de marchand tanneur), sans compter les trois enfants qui sont morts à l'âge tendre, les deux ont consacré leur vie à Dieu : la fille Angélique (née en 1720), qui était entrée chez les ursulines, mais étant devenue folle elle est morte au couvent à l'âge de vingt-huit ans ; et le benjamin Didier-Pierre (né en 1722), qui est

\author{
4 Versini 1988, 16. \\ 5 Cassirer 1983, 153. \\ 6 Ehrard 1977, 169. \\ 7 Viala 2015, 355. \\ 8 Cottret 1991, 437. \\ 9 «La grâce détermine le chrétien à agir ; la raison détermine le philosophe ». Dumarsais 1765, 509. \\ 10 Diderot 1995, 509. \\ 11 Wilson 1985, 11.
}


devenu chanoine de la cathédrale de Langres. ${ }^{12}$ Les deux autres enfants des Diderot sont le fils âné Denis (né en 1713), le futur encyclopédiste, et la fille Denise (née en 1715), que son grand frère a appelée sœurette et l'a surnommée Socrate femelle.

À l'âge de treize ans, le futur philosophe athée obtient le droit au titre d'abbé lorsque l'évêque de Langres lui donne la tonsure. Peu après, influencé par ses maîtres du collège, il envisage de devenir jésuite. Si le jeune Diderot avait eu réellement l'intention d'entreprendre une carrière ecclésiastique, cela n'aurait pas été parce qu'il avait une vocation. Comme le rappelle Arthur Wilson, les abbés, assurés des bénéfices ecclésiastiques garantissant une vie aisée, étaient des figures marquantes du paysage social du XVIII ${ }^{\mathrm{e}}$ siècle. Or, en tant que prêtre, Denis aurait pu profiter à la fois de la sécurité et des plaisirs de l'érudition. ${ }^{13}$

Bien que Denis fût familier avec les concepts théologiques et la doctrine chrétienne, nous ne pouvons prétendre que ces études théologiques fussent réellement approfondies. Recourons encore une fois au dix-huitiémiste américain constatant que le savoir théologique de Denis était « souvent emprunté » et ses arguments « trop souvent personnels » et que «plus nous examinons ses écrits, moins nous pensons justifiable d'y voir une preuve catégorique d'études [théologiques] très poussées ». ${ }^{14}$ Denis Diderot n'est devenu ni prêtre ni théologien, mais, en tant que philosophe des Lumières et encyclopédiste, voire penseur critique, il a de même traité des questions concernant la métaphysique de la Foi et la morale chrétienne, la politique de l'Église et la pratique religieuse. Il a prêché la trinité philosophique - le Vrai, le Bon et le Beau, en les trouvant valoir mieux que la Sainte Trinité de la théologie catholique, dont le serviteur dévoué était son frère cadet.

Denis Diderot est donc devenu philosophe au sens spécifique de ce mot au XVIII ${ }^{\mathrm{e}}$ siècle français, défini dans l'article encyclopédique de Dumarsais comme « un honnête homme qui agit en tout par raison, et qui joint à un esprit de réflexion et de justesse les mœurs et les qualités sociables ». ${ }^{15}$ Appelé désormais Le philosophe, Denis était considéré comme « chef de la secte philosophique », hostile à la secte des dévots à laquelle appartenait son frère. Comme DidierPierre ne suivait que la lumière du Ciel et Denis suivait les lumières de la raison, il va de soi que l'abbé Diderot reprochait au philosophe Diderot d'être impie, alors que le philosophe reprochait à l'abbé son fanatisme religieux. Selon Arthur Wilson, chacun de deux frères déplorait les opinions de l'autre tout en entretenant « une sorte d'affection obstinée totalement dépourvue de respect mutuel $\gg .{ }^{16}$ Le présent article le confirmera.

\section{L'abbé Diderot, lecteur du philosophe Diderot}

Les deux frères se sont confrontés dès débuts de leurs carrières opposées. Le philosophe Diderot, âgé de 32 ans, a envoyé sa première publication à l'abbé Diderot, âgé de 23 ans. Il s'agissait de la traduction de l'ouvrage Inquiry concerning Virtue, or Merit du penseur anglais Shaftesbury. À l'époque, il allait de soi que le traducteur prenait la liberté de corriger le texte originel, de l'élargir dans une traduction plutôt adaptée que littérale, de s'y imposer, en quelque sorte, en co-auteur. Le jeune philosophe a dédié sa traduction de l'Essai sur le mérite et la vertu (1745) au jeune abbé. L'auteur a commencé son épître par une phrase significative annonçant le

12 Didier-Pierre a étudié la théologie à Langres, puis il a fait son droit à Paris. De retour à Langres, il est devenu secrétaire de l'évêché, ensuite prieuré de Rimaucourt. À l'âge de cinquante ans, il a été nommé chanoine de la cathédrale de Langres. Marcel 1915, 607.

13 Wilson 1985, 18, 31.

14 Ibid., 30.

15 Dumarsais 1765, 510.

16 Wilson 1985, 13. 
ton de toute la dédicace de huit pages : «Oui, mon frère, la religion bien entendue et pratiquée avec un zèle éclairé, ne peut manquer d'élever les vertus morales ». ${ }^{17}$

En mentor, Denis enseigne à Didier-Pierre les principes de la vraie religion, étant loin de la folie nommé barbarie et plus proche de l'esprit philosophique résidant dans la raison. Car la philosophie ne signifie pas forcément l'impiété, tout comme la religion n'aboutit pas forcément au fanatisme ; «mais du fanatisme à la barbarie, il n'y a qu'un pas ». ${ }^{18}$ La barbarie dont parle Diderot est de l'ordre religieux, car l'autorité que l'Église a obtenue par les lois lui permet d'interdire des livres jugés impies, et, ce qui prouve les guerres de religions au $\mathrm{XVI}^{\mathrm{e}}$ siècle, lui permet d'inciter la moitié de la nation à se baigner dans le sang de l'autre moitié pour soutenir la cause de Dieu, « comme s'il fallait cesser d'être homme pour le montrer religieux ». ${ }^{19}$ Déiste au début de sa carrière de littérateur, Denis approuve qu'il n'y ait " point de vertu sans religion, point de bonheur sans vertu », et annonce ces deux vérités approfondies dans ses réflexions que l'utilité commune l'a fait écrire. L'Essai sur le mérite et la vertu se présente donc comme un antidote destiné à réparer chez Denis « un tempérament affaibli » et à entretenir chez Didier-Pierre « des forces encore entières ». ${ }^{20}$ C'est dans cet esprit que l'aîné a offert au cadet l'ouvrage traduit comme « le présent d'un philosophe et le gage de l'amitié d'un frère ». ${ }^{21}$

Roland Mortier estime que le jeune philosophe a écrit cette dédicace pour s'assurer de ne pas heurter sa famille par cet ouvrage, mais qu'il devait se rendre compte que cet éloge aurait pu être ressenti comme un affront. ${ }^{22}$ Le prouve la réaction du jeune abbé. En quatorze pages, Didier-Pierre fait des commentaires relatifs à l'épître dédicatoire, aux réflexions de Denis et au texte de Shaftesbury. En méprisant toute philosophie qui ne conduit pas à Dieu et en se faisant gloire d'être chrétien et catholique, l'abbé réfute l'épitre dédicatoire et juge que la traduction du texte de Shaftesbury est mauvaise et que le style louche et obscur empêche le lecteur de saisir bien l'esprit de l'auteur anglais. ${ }^{23}$ Mortier, qui a présenté les Réflexions de Didier-Pierre sur l'Essai sur le mérite et la vertu, conclut que, par ses griefs, l'abbé Diderot rend le philosophe Diderot « doublement coupable dès ses débuts, à la fois comme traducteur et comme penseur ». ${ }^{24}$

Dans le cas de Denis Diderot, Lucien Nouis a renversé la perspective du concept de fraternité élective, à savoir de l'idée traditionnelle du lecteur comme semblable, comme frère d'élection, au profit de la thèse du lecteur comme frère de sang. Ce théoricien croit que l'abbé Diderot hantait le philosophe Diderot dans les marges du texte comme son lecteur désiré, sinon effectif. Le philosophe ne pouvait parler de tolérance qu'en prenant en compte la présence complexe et dérangeante de son frère, affirme Nouis, et c'est la raison pour laquelle Denis a inclus Didier-Pierre en tant que lecteur et destinataire. ${ }^{25}$ Pour ce diderotien français, l'Épître dédicatoire de l'Essai sur le mérite et la vertu a « une certaine performativité du texte »: le philosophe a signé son texte doublement, à la fois en tant que philosophe et en tant que frère, et le jeune abbé, s'étant laissé prendre au jeu de la dédicace, est devenu l'otage du texte qui l'a mis en scène. ${ }^{26}$ Ayant été mentionné, Didier-Pierre a été obligé d'y répondre, car le silence même

17 Diderot 1745, III.

18 Ibid., IV.

19 Ibid., VIII.

$20 \mathrm{Ibid}$., IX.

21 Ibid., IX-X

22 Mortier 1991, 21.

23 Ibid., 39.

24 Ibid., 23.

25 Nouis 2009, 59.

26 Ibid., 63. 
aurait pu être interprété comme une réponse. Ainsi, le dialogue fraternel a-t-il pris une tournure publique. ${ }^{27}$

Le théâtre diderotien a de même causé la querelle entre les deux frères. Comme Le Fils naturel, paru en 1758, a chagriné l'abbé, l'auteur était prêt à faire une seconde édition revue suivant les griefs de son frère. Le philosophe a tenté de rassurer l'abbé qu'il préférait la tranquillité de son frère à la gloire littéraire et que l'approbation de tout l'univers sur une comédie n'équivalait pas à un moment de la peine fraternelle. Mais, après ces doux mots qu'il avait adressés à son frère dans la lettre du 29 novembre 1757, le philosophe a ôté pratiquement toute possibilité de corriger son ouvrage : «En attendant, mon cher ami, méfiez-vous de vos lumières en ce genre, et soyez convaincu que vous ne m'objecterez pas grand-chose dont je ne vous montre sur-le-champ des exemples dans des ouvrages contre lesquels on n'a jamais rien dit. $»^{28}$

L'abbé a fait des commentaires non seulement sur Le Fils naturel, mais aussi sur le traité théorique les Entretiens sur Le Fils naturel. En premier lieu, l'abbé a réfuté le rôle d'enseignement moral du théâtre. Les spectacles incitent l'homme au vice et au péché, et non à la vertu. L'abbé trouve que les personnages du Fils naturels sont d'une fausse vertu. Il se sent visé par des mots que les personnages prononcent dans la pièce, tels que préjugés, opinions monstrueuses, fanatisme. Ainsi, lorsque Constance se complaint-elle de connaître «les maux que le fanatisme a causés, et ceux qu'il en faut craindre » et se réjouit-elle que le siècle s'est éclairé et la raison s'est épurée, l'abbé y reconnaît cet odieux propos de Voltaire, qui est l'homme sans religion. ${ }^{29}$ L'abbé Diderot reproche au philosophe Diderot de ne reconnaître aucune autorité infaillible dans la société : «S'il en reconnaissait quelqu'une sur la terre, vous penseriez qu'il donnerait la préférence à l'Église ou à quelque corps de gens éclairés, sages et vertueux, mais vous vous trompez; De Voltaire et le Dieu auquel il croirait. $»^{30}$

La théorie de la dramaturgie que Denis a élaborée dans ses Entretiens sur Le Fils naturel est inacceptable pour Didier-Pierre, car le dramaturge prêche que l'ouvrage ne vaut rien dès qu'il est décent. L'abbé nie un lien de parenté entre les dévots et les poètes et les acteurs, que le philosophe a rassemblés dans « cette troupe enthousiaste et passionnée » qui sentait vivement et réfléchissait peu. ${ }^{31}$ La théorie préromantique du Second Entretien, attribuant aux génies et aux artistes une forte sensibilité, a offensé l'abbé Diderot, mécontent de voir de vrais dévots dans une compagnie des gens vicieux. L'abbé remarque que Dorval se fait gloire d'être esprit fort, à savoir « de ne rien croire, de n'avoir point de religion », ${ }^{32}$ et il considère comme un blasphème la prétention de Dorval que le christianisme est un obstacle à être honnête homme et bon citoyen. Malgré tous ces griefs de l'abbé, le dramaturge n'a pas corrigé son texte.

\section{La querelle épistolaire}

Comme l'a remarqué Flore Villemin, les échanges épistolaires entre les deux frères étaient rares et ne se justifiaient que par la nécessité de résoudre des affaires de famille. ${ }^{33}$ Chronologiquement les deux premières lettres que le philosophe a adressées à l'abbé témoignaient déjà des conflits insurmontables entre eux. Dans la première lettre, en date de 14 juillet 1756, le philosophe supplie l'abbé de ne pas se lancer dans une longue contestation avec

\section{Ibid., 59.}

28 Diderot 1997, 70.

29 Perol 1991, 41.

30 Ibid., 47.

31 Ibid., 42.

32 Ibid., 44.

33 Villemin 2011 : 53. 
le chevalier Pierre-Paul de Piolenc, son rival pour l'abbaye de Rimaucourt. «Et surtout ne plaidez pas », le conjure le philosophe, car « un honnête ecclésiastique comme vous a quelque chose de mieux à faire $» .{ }^{34}$ Douze jours plus tard, le philosophe reproche à l'abbé d'avoir réagi, dans sa réponse épistolaire, en plaideur et en fanatique. ${ }^{35}$ Denis supplie Didier-Pierre d'arrêter les procédures contre le chevalier de Piolenc et lui promet de s'engager lui-même dans des négociations. Après cinq ans, l'affaire a été terminée en faveur de l'abbé Diderot. Seize ans plus tard, au bout d'une longue période des malentendus fraternels, dans sa fameuse lettre d'adieux (du 13 novembre 1772), tout en appelant son frère méchant, infâme et le plus vil de des hommes, le philosophe lui reproche d'être ingrat pour avoir oublié que son frère l'avait aidé à obtenir l'abbaye de Rimaucourt.

Lorsque le philosophe mentionne l'abbé dans son échange épistolaire avec les autres personnes, il le dénomme ce maudit saint en ajoutant proverbialement «malheur à la famille dans laquelle il y aura un saint ! », ${ }^{36}$ ou encore le saint prêtre en ajoutant que son frère n'a pas encore fait tout le mal qu'il a à faire, mais qu'il est en bon train. Aux yeux du philosophe l'abbé est « le plus vil, le plus injuste, le plus despote et le plus intéressé des hommes ». ${ }^{37}$

Lorsque les enfants de Didier Diderot se disputaient au sujet de la succession de leur feu père, cela a provoqué des relations tendues non seulement entre les deux frères mais aussi entre Denis et sa sœurette. Le philosophe a accusé sa sœur de tenir la part de l'abbé qui l'avait persuadée que c'était « agréable à Dieu de haïr son frère ». ${ }^{38}$ Deux ans plus tard, dans sa lettre à Denise, en date de 23 mars 1770, le philosophe se rassure d'avoir tout fait pour marquer sa tendre amitié à son frère et à sa sœur, et que ces deux lui ont répondu froidement. Le 4 mai 1770, le philosophe constate toujours qu'il est bien honteux que deux frères soient fâchés l'un contre l'autre. Après ces lettres envoyées à sa sœur Denise, le philosophe a écrit à l'abbé pour se défendre de la calomnie de ses ennemis ${ }^{39}$ et pour nier le prosélytisme dont l'abbé l'avait accusé ${ }^{40}$.

Cette lettre, datée le 24 mai 1770, était la première après un long silence de dix ans entre les deux frères. La fameuse lettre sur la tolérance, du 29 décembre 1760, qui est le sujet de notre analyse dans le chapitre suivant, a probablement été la cause de cette rupture de dix ans, bien que notre épistolier se prononce de ne pas en connaître les raisons. ${ }^{41} \mathrm{Il}$ a demandé à l'abbé de lui expliquer par quel motif il s'était tenu si longtemps éloigné de son frère et de sa nièce. Il a de même écrit à Denise en affirmant que c'était leur frère cadet qui s'était brouillé avec lui sans aucune raison. En août 1770, le philosophe est parti pour Langres dans le dessein de se concilier avec son frère. L'abbé a demandé au philosophe la promesse de ne plus écrire contre la religion. Déçu, le philosophe est rentré à Paris. Une nouvelle tentative du philosophe de se rapprocher de

\section{Diderot 1997, 59.}

35 « Si ce sont là les deux qualités que donne votre religion, je suis très content de la mienne, et j'espère n'en point changer ». Lettre du 26 juillet 1756 . Ibid.

36 Lettre à Sophie Volland, du 21 novembre 1760. Ibid., 321.

37 Lettre à sa sœur Denise, du 29 mai 1768. Ibid., 829.

$38 \mathrm{Ibid} ., 830$.

39 « Mon frère, je ne suis point à l'abri des imputations calomnieuses. On m'attribuera des ouvrages que je n'aurai point faits ; des propos que je n'aurai point tenus ; mais j'espère que vous ajouterez foi plutôt à la parole d'un frère vrai, homme de bien, qui n'a aucun intérêt à vous dissimuler la vérité, [...] qu'à des bruits populaires qui ne signifient rien. »Ibid., 1012.

40 «Écoutez bien ce que je vais vous dire. Je n'ai point et je n'eus jamais la folie du prosélytisme. Je pense pour moi et je pense pour moi seul. Je laisse les autres dans leurs sentiments. »Ibid.

41 Benoît Melançon explique la rupture entre les frères par trois causes : l'impiété de l'aîné ; le mariage de la fille du philosophe avec Caroillon de Vandeul, soupçonné d'irréligion ; des raisons économiques liées à l'héritage laissé par Didier Diderot à ses trois enfants, Denis, Denise et Didier-Pierre. Melançon 1996, 161, n. 75. 
son frère a échoué : l'abbé a refusé de donner sa bénédiction pour le mariage de MarieAngélique, la fille du philosophe. Didier-Pierre a expliqué sa démarche dans ses deux lettres écrites à la fin d'août. Dans la première, il apprend à Denise que « le peu de religion et le défaut de vertus sociales » du futur époux de Marie-Angélique l'empêchent de bénir cette alliance. ${ }^{42}$ L'abbé rappelle sa sœur qu'il a demandé à Denis, il y a dix ans, de désavouer tous les écrits qu'il avait faits contre la religion et de promettre qu'il vivrait désormais en chrétien. Dans la seconde lettre, l'oncle reproche à sa nièce d'avoir été élevée « dans une tolérance inexcusable pour une véritable chrétienne » et de vouloir épouser un antichrétien. L'abbé est bel est bien ferme : si le mariage a lieu, Marie-Angélique n'est plus sa nièce comme Denis n'est plus son frère, pour le même motif de religion. ${ }^{43}$

Ces deux lettres de l'abbé sont en date d'août 1772. L'automne a marqué la rupture définitive entre les deux frères dont témoignent quatre dernières lettres échangées : les deux écrites par le philosophe et les deux par l'abbé. Tout d'abord, le philosophe écrit à son frère le 25 septembre, et en le dénommant un bigot lui reproche d'avoir prêché à Marie-Angélique la haine de son père. Il lui recommande de lire dans l'Encyclopédie l'article «Intolérance », en le conseillant de se rendre à la société et de revenir dans sa famille. Le philosophe affirme d'avoir rempli les conditions de l'abbé, de ne jamais rien publier contre la religion, et cependant, il a perdu son frère. En lui annonçant que c'est la dernière lettre qu'il recevra, le philosophe finit son sermon fraternel : «Les sophismes disparaissent au lit de la mort. C'est alors que tu te verras mauvais prêtre, mauvais citoyen, mauvais fils, mauvais frère, mauvais oncle, méchant homme. ${ }^{44}$ Toutefois, ce n'était pas la dernière lettre, car le philosophe serait provoqué par la riposte de l'abbé.

Dans la lettre écrite fin octobre ou début novembre 1772, l'abbé en veut au philosophe de lui avoir répondu par l'injurier, ce qui ne l'étonne point, car son frère excelle dans ce genre de défense. Il croit que M. le philosophe s'accommode de tout, excepté de ce qui contrarie ses idées antichrétiennes. L'abbé a cessé de réagir aux ouvrages que son frère écrivait contre la religion, car les lettres seules du philosophe suffisaient, étant écrites dans l'esprit de l'incrédulité et de l'impiété. En s'adressant à M. l'Encyclopédiste, l'abbé lui reproche de prêcher que le vice et la vertu, le bien et le mal, ne sont que des mots synonymes et l'accuse de n'avoir jamais été reconnaissant envers son frère. S'il se voit mauvais prêtre, c'est pour n'avoir pas soutenu avec assez de vigueur les intérêts de Dieu devant son frère impie ; ou mauvais citoyen, c'est pour n'avoir pas fait connaître son aîné plutôt comme une peste dans l'État ; ou mauvais frère, c'est pour avoir au début trop de condescendance à l'égard de son aîné ; ou mauvais fils, c'est pour avoir laissé ignorer à leurs parents que Denis s'était marié contre leur consentement; ou mauvais oncle, c'est pour n'avoir pas arraché sa nièce à son père impie afin de lui faire donner une éducation chrétienne. ${ }^{45}$ Le philosophe riposte par une longue lettre, la dernière qu'il écrira à son frère. Nous pouvons la compter parmi deux lettres majeures du philosophe ayant le même sujet - l'intolérance religieuse.

\section{Sur l'intolérance religieuse}

À travers l'analyse précédente de la correspondance familiale, nous avons pu conclure que même une affaire de famille en cours faisait place aux reproches mutuels tournant toujours autour du même sujet - la religion, ou plus précis l'intolérance. Les deux lettres majeures que le

42 Lettre du 27 août 1771. Perol, Chouillet 1990, 16.

43 Lettre sans date [fin août 1772]. Ibid., 18.

44 Diderot 1997, 1129.

45 Perol, Chouillet 1990, 27. 
philosophe Diderot a envoyées à l'abbé Diderot représentent de vrais traités polémiques que nous pourrions les intituler Lettres sur l'intolérance religieuse. La première de ces lettres porte la date du 29 décembre 1760, et elle sera quasiment reproduite en 1765 dans l'article « Intolérance » du tome VIII de l'Encyclopédie. ${ }^{46}$

Denis écrit à son frère en condamnant la pratique religieuse imprégnée de l'intolérance envers les autres qui osaient penser, croire ou agir autrement que l'église catholique. Pour lui, impie est tout moyen «qui excite la haine, l'indignation et le mépris », «qui tient à des vues intéressées », « qui tendrait à armer les nations et tremper la terre de sang $» .{ }^{47}$ En conseillant à son cadet l'instruction, la persuasion et la prière comme les seuls moyens d'étendre la religion, l'aîné lui apprend qu'il faut éclairer la conscience et non la contraindre. ${ }^{48}$ Selon le philosophe, la voix de l'humanité n'est pas celle du persécuteur qui frappe, mais celle du persécuté qui se plaint. Pour soutenir ses arguments, Denis recourt aux Évangiles, aux Actes des Apôtres, aux épitres de saint Pierre, aux textes de Saint Augustin et aux messages du IV ${ }^{\mathrm{e}}$ concile de Tolède en 633. Il rappelle Didier-Pierre que les Évangiles nous enseignent de laisser le jugement à Dieu.

De ces deux Lettres sur l'intolérance religieuse, comme je les ai désignées, ces't avec la seconde, signée le 13 novembre 1772, que le philosophe met le point final à l'échange épistolaire avec son frère. Denis y réagit à la lettre précédente de l'abbé, écrit en octobre ou en novembre $1772 .{ }^{49}$ En répondant au « mauvais frère et mauvais oncle » qui a renié son frère et sa nièce, l'aîné reproche à son cadet, pour mille et unième fois, sa conduite injuste, le caractère orgueilleux et la fureur de son fanatisme. Le philosophe se permet d'enseigner à l'abbé la doctrine de l'Évangile et le conseille de se prosterner et de frapper son front contre la pierre pour tâcher d'obtenir le pardon du Ciel. Le philosophe finit sa leçon sur la tolérance et sur la miséricorde chrétienne par - «plus de lettres à recevoir, plus de réponses à faire ». ${ }^{50}$

Toutefois, l'abbé a répondu par la lettre du 14 décembre 1772, que le Philosophe renverrait à l'expéditeur sans l'ouvrir. Dans cette lettre sans salutation et sans signature, écrite d'un ton froid et dictée par une ironie amère, Didier-Pierre lance des attaques contre l'Encyclopédie, considérée comme impie par des gens respectant les bonnes mœurs, et il se déclare heureux d'être pris comme fou par les hommes de la secte philosophique. ${ }^{51}$ Mais Denis Diderot a rompu ce cercle vicieux de l'échange épistolaire en renvoyant la lettre sans la lire. ${ }^{52}$ Comme l'a vu Flore Villemin, la communication entre les deux frères, de difficile, est devenue

46 La lettre est rendue publique pour la première fois dans la Correspondance littéraire de Grimm (1761). Une seconde distribution s'est déroulée sous une forme très proche dans l'article «Intolérance » publié en 1765 dans le tome VIII de l'Encyclopédie. Nouis 2009, 66.

47 Diderot 1997, 336.

48 Ce même sermon philosophique sera reproduit dans l'article encyclopédique cinq ans plus tard. L'article commence par la définition du mot intolérance signifiant communément «cette passion féroce qui porte à haïr et à persécuter ceux qui sont dans l'erreur ». Le philosophe distingue deux sortes d'intolérance qui vont de pair : l'intolérance civile n'est que la conséquence juridique de l'intolérance ecclésiastique. Ibid., 843.

49 Bien que le philosophe épistolier ait juré que sa lettre précédente, du 25 septembre 1772, serait la dernière adressée à l'abbé, après un mois et demi, suit une longue lettre (onze pages imprimées dans la Correspondance établie par Laurent Versini) dans laquelle l'aîné, en considérant son cadet comme ingrat, infâme, méchant, bête, le dénomme « un monstre infernal », « un étrange chrétien », « une véritable éponge à scandale », « un enragé persécuteur », et «le plus vil des hommes ».

50 Ibid., 1152.

51 J'ai consulté la dernière lettre de l'abbé dans la version que Lester Crocker a publiée dans son ouvrage sur Diderot. Crocker 1955, 371-372.

52 Denis renvoie la lettre, le 17 ou 18 décembre, avec la notice suivante : «M. l'abbé, si j'étais sûr de retrouver mon frère dans cette lettre, je l'ouvrirais et je ne la lirais pas sans verser des larmes de joie. Mais, j'aime mieux vous la renvoyer toute cachetée, et m'épargner deux peines ; l'une, d'entendre et l'autre de répondre des choses déplaisantes. » Diderot 1997, 1152. 
impossible, et le philosophe a préféré désormais le silence à l'éprouvant dialogue de sourds. ${ }^{53}$ Suivent douze ans de silence, jusqu'à la mort du philosophe en 1784. L'abbé est mort trois ans plus tard, en 1787.

\section{En guise de conclusion}

Selon Flore Villemin, la figure du philosophe se construisait avec le temps en opposition à la figure du dévot, et ces deux figurent constituent le pendant l'une de l'autre. Ainsi, l'abbé s'impose-t-il comme « une sorte de figure prototype du dévot, hantant l'écriture du philosophe $» .{ }^{54}$ Pour Denis, Didier-Pierre fait preuve de la plus grande intolérance religieuse. Le philosophe oppose son éthique civile à la morale religieuse de l'abbé. Non seulement que la religion ne va pas nécessairement de pair avec la vertu, mais bien au contraire : la religion va souvent avec l'immoralité. ${ }^{55}$ Quant à Didier-Pierre, il avait honte d'un frère irréligieux séparant la morale du christianisme. Comme un dévot revendique la subordination de la raison à la foi, il va de soi que l'abbé Diderot n'était pas séduit par le propos du philosophe ayant cherché à le convaincre «de délaisser une fausse fraternité chrétienne pour la remplacer par une fraternité universelle $» .^{56}$

Lucien Nouis constate que Didier-Pierre représentait pour le philosophe son lecteur idéal, et que, pour le philosophe, cesser de prôner la tolérance à l'abbé intolérant, ce serait cesser de philosopher. Toutefois, tous les deux sont restés fidèles à leurs convictions opposées, et le débat a fini par la rupture définitive de la relation fraternelle. Le silence entre eux est resté jusqu'à la mort du philosophe, mais leur débat n'a cessé de susciter l'intérêt des chercheurs, non seulement de ceux au $\mathrm{XX}^{\mathrm{e}}$ siècle, mais aussi bien de ceux au début du XXI $\mathrm{XX}^{\mathrm{e}}$ siècle, tels que Nouis (2009) et Villemin (2011). Le présent article a essayé de reconsidérer le discours argumentatif de Denis et de Didier-Pierre, deux débatteurs qui avaient des points de vue opposés au sujet de la morale et de la tolérance, à savoir de la philosophie et de la religion.

\section{Sources}

Crocker, Lester G. (1955): The Embattled Philosopher. London: Neville Spearman.

Diderot, Denis (1745) : «Épitre dédicatoire: À mon frère ». In : Principes de la philosophie morale, ou Essai de M. $S^{* * *}$ sur le mérite et la vertu. Avec réflexions. Amsterdam : Chez Zacharie Chatelain, III-X.

Diderot, Denis (1994) : «Irréligieux ». In : Euvres : Philosophie, éd. Laurent Versini. Paris : Robert Laffont, 467-468.

Diderot, Denis (1765) : «Intolérance». In : Encyclopédie, t. VIII, 843. <https://artflsrv03.uchicago.edu/philologic4/encyclopedie1117/navigate/8/3473/>. 3. 5. 2021.

Diderot, Denis (1995) : «Observations sur le Nakaz». In : Euvres : Politique, éd. Laurent Versini. Paris : Robert Laffont, 501-578.

Diderot, Denis (1997): Euvres: Correspondance. Sous la direction de Laurent Versini. Paris : Robert Laffont.

53 Villemin 2011, 89-90.

54 Ibid., 53.

55 Dans son article «Irréligieux », publié dans le tome VIII de l'Encyclopédie (1765), Diderot, en bon disciple de Bayle, souligne qu'il ne faut pas confondre l'immoralité et l'irréligion : «La moralité peut être sans la religion; et la religion peut être, est même souvent avec l'immoralité. » Diderot 1994, 467-468.

56 Nouis 2009, 65 . 
Dumarsais, César Chesneau (1765) : «Philosophe ». In : Encyclopédie, t. XII, 509511. <https://artflsrv03.uchicago.edu/philologic4/encyclopedie1117/navigate/12/2064/>. 18. 4. 2021.

Marcel, Le Chanoine (1915) : «Le frère de Diderot». Comptes rendus. In : Revue d'Histoire littéraire de la France, $\mathrm{n}^{\mathrm{0}}$ 3-4. Paris : Presses Universitaires de France, 607-609.

Mortier, Roland (1991) : «Didier Diderot lecteur de Denis : ses Réflexions sur l'Essai sur le mérite et la vertu. » In : Recherches sur Diderot et sur l'Encyclopédie, $\mathrm{n}^{\circ} 10$. Paris, 2139.

Perol, Lucette et Anne-Marie Chouillet (1990) : «L'abbé Diderot et le philosophe, échange de lettres présenté ». In : Recherches sur Diderot et sur l'Encyclopédie, ${ }^{\circ}$ 9. Paris, 1040 .

Perol, Lucette (1991) : «Didier Diderot lecteur de Denis : ses Réflexions sur le Fils naturel ». In : Recherches sur Diderot et sur l'Encyclopédie, $\mathrm{n}^{\circ} 11$. Paris, 33-47.

\section{Références bibliographiques}

Cassirer, Ernest (1983) : La philosophie des Lumières. Paris : Fayard.

Cottret, Monique (1991) : «Lumières (Siècle des) ». In : Encyclopédie de la culture française, dir. Bernard Willerval. Paris : Eclectis, 437-438.

Ehrard, Jean (1977) : « Diderot » (Chapitre IV). In : Robert Mauzi et Sylvain Menant, Littérature française, Le XVIII ${ }^{e}$ siècle, II, 1750-1778, collection dirigée par Claude Pichois. Paris : Arthaud, 169-200.

Melançon, Benoît. (1996) : Diderot épistolier. Contribution à une poétique de la lettre familière au XVIII siècle. Québec : Éditions Fides, 1996.

Nouis, Lucien (2009) : «Diderot et son frère ». In : Diderot Studies, vol. 31. Genève : Librairie Droz, 57-70.

Versini, Laurent (1988) : Le XVIII ${ }^{e}$ siècle, Littérature française. Nancy: Presses Universitaires de Nancy.

Viala, Alain (2015) : Une histoire brève de la littérature française. L'Âge classique et les Lumières. Paris : Presses Universitaires de France.

Villemin, Flore (2011) : La réfutation chez Diderot : le discours du philosophe dans la polémique religieuse. Thèse de doctorat. Université Nancy 2.

Wilson, Arthur (1985) : Diderot, sa vie et son œuvre. Paris : Laffont-Ramsay. 


\section{Нермин Вучељ}

\section{ФИЛОСОФ ДИДРО ПРОТИВ СВЕШТЕНИКА ДИДРОА}

Рад нуди анализу критичке полемике коју су браћа Дидро, философ Дени и свештеник Дидије-Пјер, водили на тему религије, морала и толеранције. Ако пођемо од хипотезе да је опат Дидро био његов жељени читалац, онда је философ Дидро, мањевише, писао своја дела предочавајући себи као циљаног реципијента сопственог брата, који је био непријатељ Енциклопедије и философије. У складу с просветитељском мисијом, философ Дидро у млађем брату види прототип задртог верника којег треба просветлити. Насупрот томе, свештеник Дидро критикује старијег брата као безбожника који неосновано одваја морал и хришћанство. Овај чланак настоји да преиспита сучељена гледишта двојице полемичара. 